\title{
An Iterative Methodolgy to Improve TV Onscreen Keyboard Layout Design through Evaluation of User Studies
}

\author{
Debatri Chatte rjee ${ }^{1, *}$, Aniruddha Sinha ${ }^{1}$, Arpan Pal $^{1}$, Anupam Bas u ${ }^{2}$ \\ ${ }^{1}$ Innovation Lab, Tata Consultancy Services Ltd., Kolkata, 700091, India \\ ${ }^{2}$ Indian Institute of Technology, Computer Science Department, Kharagpur, India
}

\begin{abstract}
Certain applications (e.g web browsing, email etc.) in Television (TV) demand for text entry by the user in a similar fashion as done in computers. User experience plays a major role in the success of such applications. This paper discusses about an on-screen keyboard with hierarchical character or symbol organization which is operated by an accompanying remote control to allow navigation with reduced number of key strokes. The keyboard has been designed for Television and set top box users. It enhances user experience by allowing users to type easily and quickly. It also reduces cost by eliminating the need for a separate physical keyboard. This work has adopted iterative design cycles, where users' feedback arecollected and analysed in each cycle, and those have led to user-driven usability imp rovement. As the main contribution in this paper, we present a methodology for evaluating different on-screen keyboard layouts for television and set-top box users. In order to do this we have extended and applied KLM-GOMS model. Lastly we have incorporated predictive text entry technique with the proposed layout. We have extended the KLM-GOMS model further to include a new parameter called dynamic mental operator(DM) which takes into account the additional cognitive load on the users while using predictive text entry techniques.
\end{abstract}

Keywords Onscreen Keyboard, KLM -GOMS, Predictive Text Entry

\section{Introduction}

The world is witnessing phenomenal convergence in terms of content, access technology and access device. Several categories of contents like voice, data and mu ltimed ia content can be converged on and accessed on the same device. Content from multiple applications including voice call, video call, television program, Internet, video conference, short message service and instant message can be accessed using a single access device which can be desktop computer, laptop computer, mobile phone, PDA, Television, Set Top Box or any suitable device.

In almost all developing countries, average Internet penetration using PCs in households is $28.7 \%$ which is significantly low compared to that of televisions which is almost $75 \%([1],[2],[3])$ as the majority of the people are from low income group and they can't afford to have a personal computer, Whereas significant majority of them have a television at home as a family entertainment consumer device.

* Corresponding author:

debatri.chatterjee@tcs.com(Deb atriChatterjee)

Published online at http://journal.sapub.org/ac

Copyright (C) 2012 Scientific \& Academic Publishing. All Rights Reserved
Hence it is useful to provide internet facility on TV through the use of suitable set top box or other enabling devices.

Television screens are usually bigger than some of the access device screens like mobiles / tablets and this makes it ideally suitable for accessing wide range of applications and content. Though a significant majority of users have a television at home, the potential of interactive television remains largely unfulfilled[4]. It is even possible for people to browse Internet from the TV through use of suitable Set Top Box or other enabling devices [24].

With the new evolving functionality in television-based home entertainment applications and internet browsing, there is an increased need to enable its users to input text through onscreen keyboards and remote control devices[5]. Traditional keyboard increases the overall cost of the system. So an effective low-cost solution is to enable users to use remote controls for text entry. Thus it is clear that along with being natural and intuitive, the interaction mechanis m should allow us er to accomp lish their desire task easily and quickly, rather than spending too much time dealing with the interface mechanism. To achieve this goal, the authors proposed a novel organization of characters and symbols for the on-screen keyboard[9] based on a mathematical foundation, which can be operated by a specially designed remote control to significantly reduce the 
number of key strokes while typing during use of the services. Thus it allows users to access and enjoy the services with less effort and in less time. After designing the keyboard a user study was conducted to evaluate and monitor the acceptance of this layout[10]. Based on the study results some modifications have been done on the layout. The on-screen keyboard layout has been evaluated using an extended KLM-GOMS[11] model. This paper mainly describes a methodology of evaluating different onscreen keyboard layouts for Television and set top boxes. In order to model the remote based operations, the standard KLM operator set has been extended by introducing new operators and by modifying existing ones. This extended KLM can therefore be applied to evaluate other interfaces of this class as well.

In this paper word prediction feature has been added to the onscreen keyboard layout. Though it appears that reduction in keystrokes increases typing speed but actually it results in higher cognitive load as we have to go through the suggestions with every keystroke[18]. In this paper we have tried to evaluate the usage of prediction along with the onscreen keyboard layout based on the cognitive load on the user. And finally, we have modified KLM-GOMS model further to, take into account the additional cognitive load. It is to be noted here that the current objective is to study the user behaviour when the word prediction is integrated with the on-screen keyboard rather than evaluating the prediction software.

The organization of this paper is as follows: Section 2 of this paper presents related works in this field and the issues faced with solutions given. It also states the issues with the present scenario of typing in TV screen. Section 3 gives the overview of the proposed system. It also contains a detail description of the extension of KLM-GOMS model used for evaluation. Section 4 details the extension of GOMS model. The user studies conducted have been detailed in section 5 . Section 6 contains the methodology for design evaluation using KLM-GOMS. Results obtained are given in section 7. It also contains some graphs indicating that the proposed keyboard layout is better than other available on- screen layouts. It also includes the results of KLM-GOMS. Section 8 contains the design updates made based on the results obtained through user study. Section 9 summarises the conclusions of this paper.

\section{Related Works and Issues}

With the new evolving functionality in television-based home entertainment applications and internet browsing, there is an increased need to enable its users to input text through remote control devices. This brings out the importance of an on-screen keyboard layout which can be used for rapid and accurate text entry[5].Traditional keyboard increases the overall cost of the system. So an effective low-cost solution is to enable users to use remote controls for text entry. Different types of remote controls can be used for this purpose. The use of traditional Digital
Terrestrial Television (DTT) remote control for text entry has already been proposed in[6].Similarly The NumpadTyper (TNT)- numeric keypad based text input method is explained in[7].An onscreen keyboard can be operated with multiple input devices, which may include a touch screen, an actual keyboard, and a computer mouse. Virtual keyboards can also be accessed using different types of remote controls, but most of the layouts are not suitable for home entertain ment context because they normally use a stylus or a QWERTY keyboard. Systematic design of virtual keyboards with auto-scan access feature for mouse disabled users has been presented in[16, 17]. This paper describes a methodology to evaluate different onscreen keyboard layouts based on extended KLM-GOMS model. The best suited onscreen keyboard layout selected isused to conduct a user study. The layout has been further modified based on the user feedback received.

\section{System Overview}

To achieve the stated goals, a system is proposed where an on-screen keyboard is displayed on the monitor of a Television, Computer or similar devices[9]. The character set is organized in blocks with each block containing up to a maximum of 4 characters (Figure 1). The characters in the on-screen is organized into blocks of up to 4 characters or symbols, or character-sets in a block. However it is possible to have fewer than 4 characters, or symbols or character-sets in a key-block for providing greater prominence to some characters, or symbols or character-sets and thus better ease-of-use. The algorithm in Figure 5 describes a method for organizing a given number of characters, symbols or character-sets into an optimum number of key-blocks organized horizontally and vertically. The number of key-blocks in horizontal direction is called columns and number of key-blocks in the vertical direction is called rows.

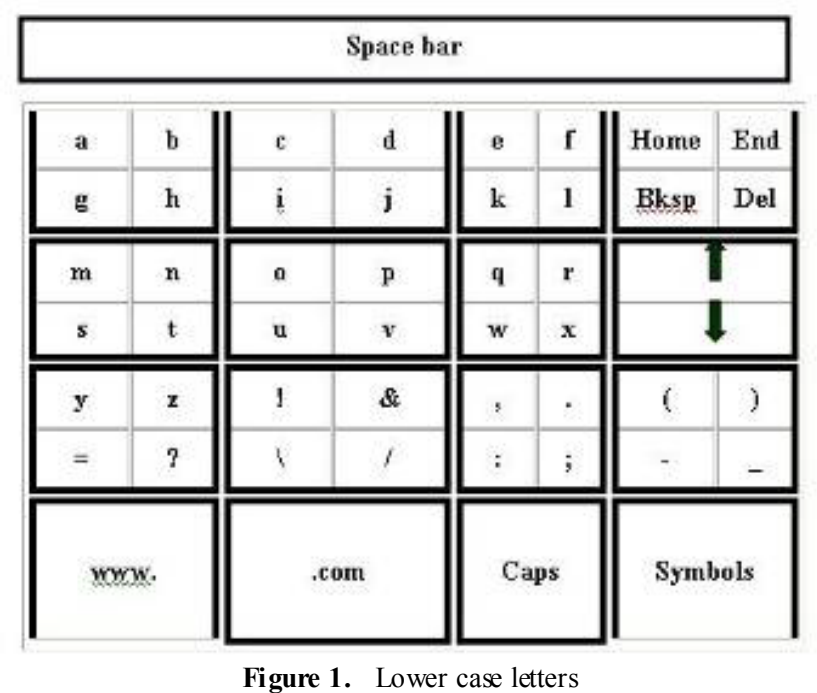

In Figure 1, the top-left key-key block following the space bar key-block contains the characters a, b, g, h. Each of the 
individual portions in a key-block is called cell. Thus the key-block above contain 4 cells and the cells contain a,b,g and $h$. A cell can contain a single character, a symbol or a set of characters. The character sets can be commonly used sequence in a given domain like "www." or ".com" in Internet.

The advantage of this kind of character-sets is that the complete character-set can be typed with a single selection reducing the typing effort even further. Instead of typing ".com" separately using 4 characters, selecting the ".com" short-cut will achieve the same result with significantly reduced key strokes. In the above-mentioned key-block the letter $\mathrm{a}$ is in the up-left cell in top-left corner, the letter $\mathrm{b}$ is in the up-right cell in top-right corner, the letter $g$ is in the down-left cell in bottom-left corner and the letter $h$ is in the down-right cell in bottom-right corner.

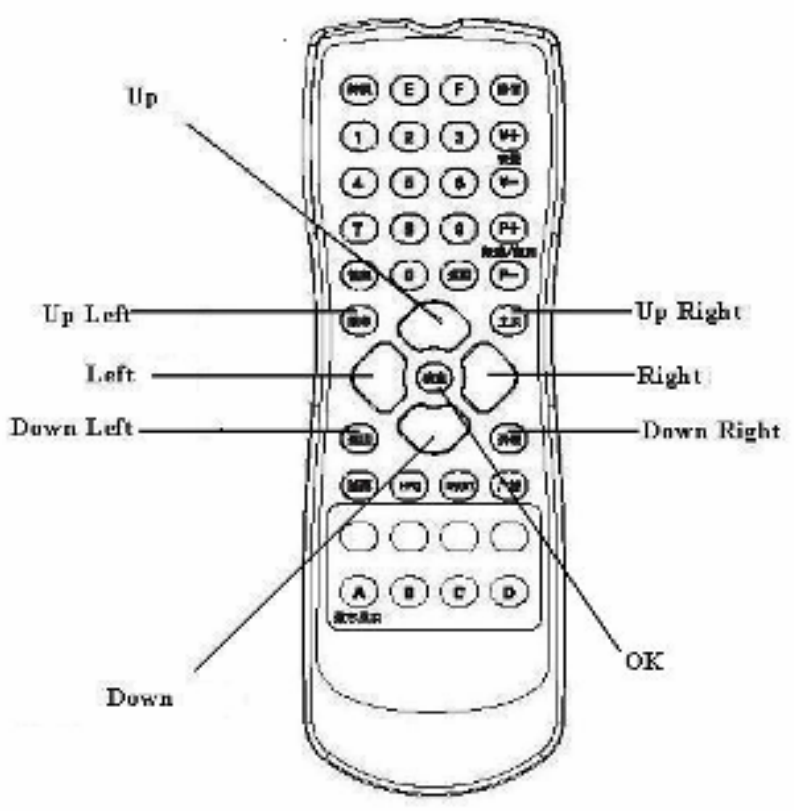

Figure 2. Accompanying Remote Control

This onscreen keyboard is operated by a remote control (Figure 2) which has among other things 9 keys for performing navigation and selection of character or symbols. The Up, Down, Left and Right keys in the remote control is used to move across the key-blocks in vertical and horizontal direction. As these 4 keys are used to navigate across key-blocks they are called Navigational keys. The remote-control also has Up Left key, Up Right key, Down Left key, Down Right key and OK key. Up Left key, Up Right key, Down Left key, Down Right key are also called diagonal arrow keys. These 5 keys are used to select one of the available character, symbols or character-sets in a key-block and hence they are called selection keys. After navigating to a key-block, Up Left key can be used to select the character, symbol or character-set in the up-left cell, Up Right key can be used to select the character, symbol or character-set in the up-right cell, Down Left key can be used to select the character, symbol or character-set in the down-left cell, and Down Right key can be used to select the character, symbol or character-set in the down-right cell. If a key-blocks has only 2 cells organized vertically, either of Up Left and Up Right key can be used to select content in upper cell and either of Down Left and Down Right can be used to select content in lower cell. a key-blocks has only 2 cells organized horizontally, either of Up Left and Down Left key can be used to select content in left cell and either of Up Right and Down Right can be used to select content in right cell. The switching between lower case letter screen, upper case letter screen, symbol screen and any other types of screens can also be achieved through specially as signed hot keys in the remote control. Thus the on-screen keyboard framework invokes multiple screens, where each screen caters to a host of inputting options. Some keys have a constant function on each screen and some keys have variable functions on various screens. The keys are thus encoded to produce multiple outputs.

Figure 3 provides flow-chart for the algorithmic representation for organizing a given numbers of characters or symbols or character-sets optimally so that number of required keystrokes is reduced.

It can be deduced from this algorith $m$ that for $T$ number of characters, a square number $\mathrm{N}$ having an even number square root and is equal to $\mathrm{T}$ or just the next square number after $\mathrm{T}$. The reason for having even number square root is that in both horizontal and vertical directions, there can be a minimum of 2 cells per key-block. The total character can be optimally organized in $\mathrm{R}$ rows and $\mathrm{C}$ columns of blocks where,

- $R=N / 2$ and $C=N / 2$ when $\mathrm{N}=\mathrm{T}$ or

- $R=(N-2) / 2$ and $C=N / 2$ or $R=N / 2$ and $C=(N-2) / 2$ when $\mathrm{N}<\mathrm{T}$

For example, for $\mathrm{T}=48, \mathrm{~N}=8$, the proposed layout gives, $\mathrm{R}=3$ and $\mathrm{C}=4$.

Due to this character layout, any character is a maximum of $(\mathrm{R}+\mathrm{C}-1)$ keystrokes away on the same screen and $(\mathrm{R}+\mathrm{C})$ keystrokes away on a different screen. $(\mathrm{R}+\mathrm{C})$ is minimum when the proposed algorithm is used to derive $\mathrm{R}$ and $\mathrm{C}$.

This particular keyboard layout was evaluated both theoretically and through user study[10],[11]. Based on the reports obtained through user study, some important modifications have been adopted in the proposed layout. For theoretical performance evaluation KLM-GOMS model has been used. In order to model the remote based operations, the standard KLM operator set has been extended in the present work. In particular, we have selected two configurations as shown inFig.4 and Fig.5, proposed in[9] besides the standard QWERTY configuration. The configurations have been compared for performance, with respect to two representative tasks, namely text entry and sending email. The results predicted from the KLM-GOMS models have been compared and verified against the results obtained from user study. 


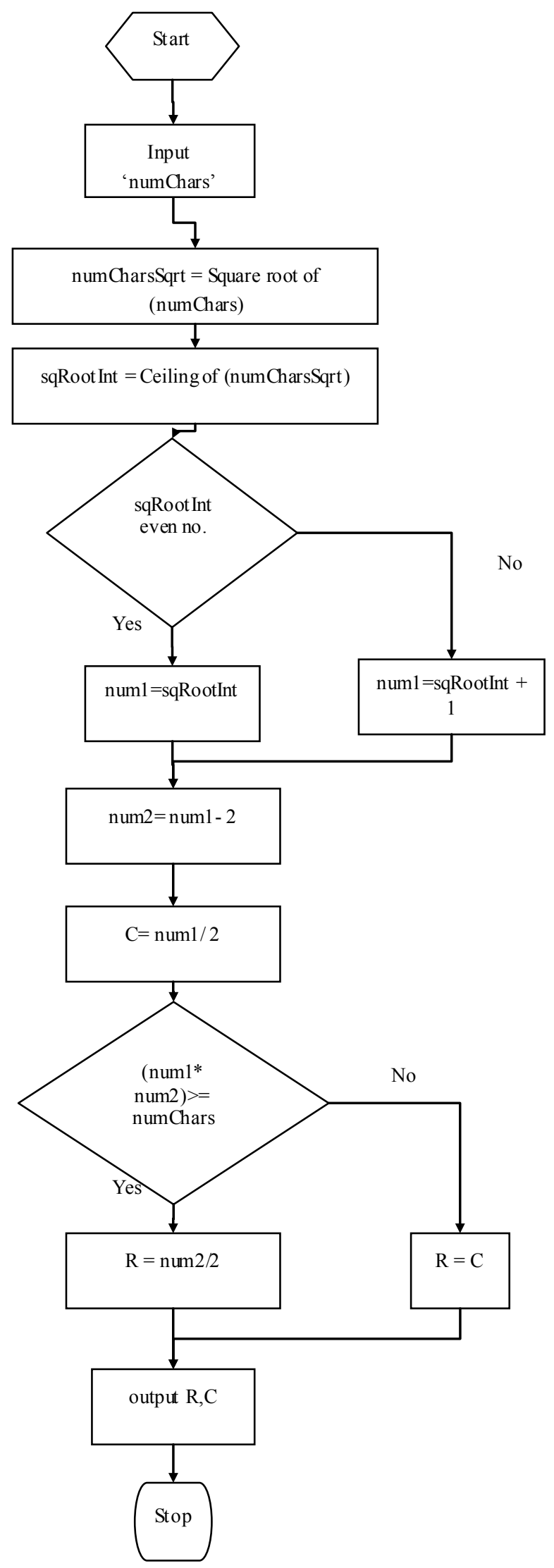

Figure 3. Flow-chart
In paper[11] we evaluated the proposed onscreen keyboard layouts based on Keystroke-level-model (KLM) and Goal-Operator-Methods (GOMS) model for a user application. A KLM consists of a number of operators. There are five basic operators which are of relevance here. These operators are listed in table 1.

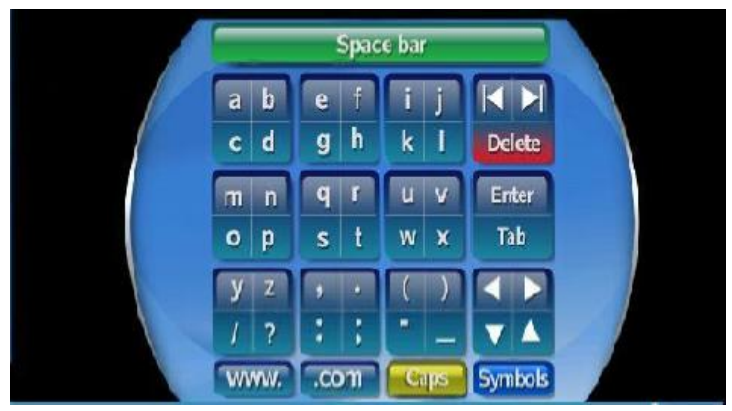

Figure 4. On-screen keyboard layout 1

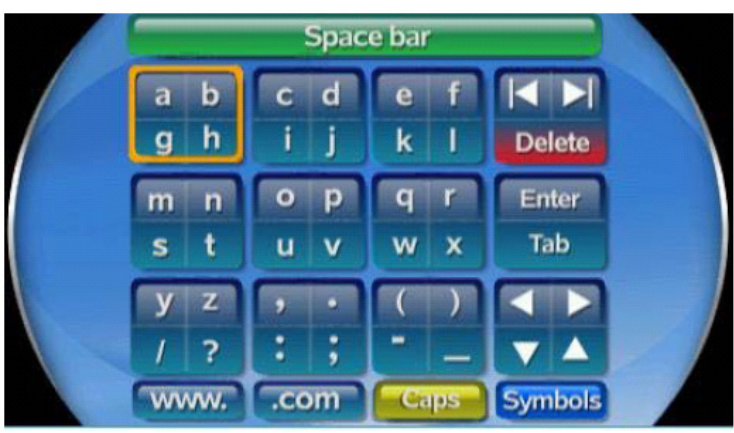

Figure 5. On-screen keyboard layout 2

Table 1. KLM-GOMS operators

\begin{tabular}{|c|c|c|}
\hline Operators & Description & Time in sec \\
\hline $\mathrm{P}$ & Finding a key and moving focus & $\begin{array}{c}1.10 \text { or } 1.73 \text { or } \\
1.77\end{array}$ \\
\hline $\mathrm{K}$ & Key or button press & 0.20 \\
\hline $\mathrm{H}$ & $\begin{array}{c}\text { Move from mouse to keyboard } \\
\text { and back }\end{array}$ & 0.4 \\
\hline $\mathrm{R}(\mathrm{t})$ & $\begin{array}{c}\text { Wait ing time for device to } \\
\text { respond }\end{array}$ & $\mathrm{t}$ \\
\hline $\mathrm{M}$ & $\begin{array}{c}\text { Mental preparat ion and thinking } \\
\text { time }\end{array}$ & 1.35 \\
\hline $\mathrm{F}$ & Finger movement & 0.22 \\
\hline
\end{tabular}

The details of all the parameters are given in [11]. The values of the operators $\mathrm{K}, \mathrm{H}$ and $\mathrm{M}$ have been adopted from original KLM model. The definition of the P operator has been modified keeping in mind the present scenario. In original KLM model, $\mathrm{P}$ operator represents pointing with a pointing device at a particular position of the screen, excluding the button press. In the present scenario, as the layouts follow a block structure(containing max 4 alphabets in each block), the action of pointing to a specific key can essentially be subdivided into two actions. One is finding the required key within the layout and the other is focusing on that particular block with left/right navigation keys of the remote control. Hence we have re-defined $\mathrm{P}$ as the total time taken in finding a key on a particular layout and moving the focus to select the block containing that particular key. To estimate the value of $\mathrm{P}$, a user study was conducted.

\section{Extended GOMS Model}


The $\mathrm{H}$ operator was found to be useful for standard QWERTY keyboard layout. The layout 1 and layout 2 were solely operated using a remote control, hence for them, the $\mathrm{H}$ operator was of no use.

A new parameter $F$ has been introduced for measuring the time required for finger movement. The justification of including this parameter has been explained in[12]. The average time of finger movement (F) in a QWERTY keyboard has been found to be $0.22 \mathrm{sec}$.

Finally word prediction feature was integrated with the onscreen keyboard layout. There is a text area above the predictive on screen keyboard layout for entering data. As the user starts entering letters, four suggestions appear on four different colour coded suggestion boxes as shown in figure 6 below.

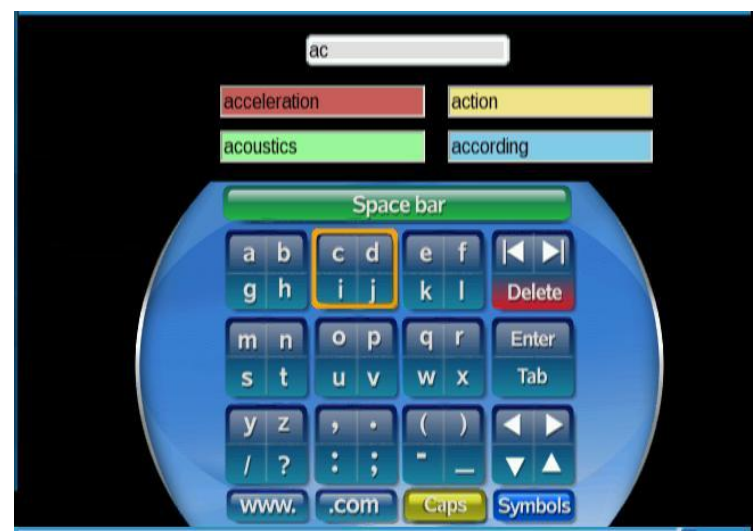

Figure 6. On-screen keyboard with predictive text entry

These suggestions change as soon as the user enters a new character. If the user selects any of the four suggested words, then that particular word is displayed in the text area. The selection of suggested words can be done by using any of the four colour keys of the remote control (Figure 2). The red button for selecting word displayed in red box, blue button for selecting word displayed in blue box and so on Once a word is selected by user, the application updates the word and calculates the frequency of its use. If a particular word is selected repeatedly, then next time that word comes first as a suggestion. Separate user study was conducted to evaluate the performance of the onscreen keyboard with word prediction. Based on the study results we have introduced a new operator for KLM-GOMS model. This operator is called dynamic mental operator and it takes into account the additional cognitive load on the user for using a word completion system. It is true that a predictive system reduces the number of keystrokes required to enter any words but at the same time it also increases the cognitive load on the user as with each keystroke, the user needs to go through the options given and choose one[18]. High keystroke savings does not indicate success of a word prediction system. For fast typists letter-only typing is the best possible method. Word prediction is best particularly for users with moderate to slow key press times[19] and the better communication rate also depends on the prediction used and on the user[21].
The KLM-GOMS equation[20] for traditional text entry of a given phrase can be written as:

Where,

$$
T=T_{h}+w\left(k_{t} T_{k}+d T_{m}\right)
$$

$T_{h}=$ homing time

$T_{k}=$ time for button press

$k_{t}=$ average number of key presses per word

$\mathrm{w}=$ number of words typed

For predictive text entry, the prediction algorith m reduces the total number of keystrokes. The algorithm we used in our system approximately reduces no. of keystrokes by 0.399 .

Hence, Effective key-press is

$$
K_{\text {eff }}=w k_{t} * 0.399
$$

Again, for predictive text entry an additional mental operator comes into play. This corresponds to the additional cognitive load for reading and selecting suggestions provided. The suggestions changes with each keystroke. Thus, equation (1) becomes,

$$
T=T_{h}+K_{e f f} \cdot T_{k}+w \cdot d \cdot T_{m}+K_{e f f} \cdot T_{d m}
$$

Where, $T_{d m}=$ additional mental operator

$=$ time for reading suggestions (dynamic mental

(DM) operator) + one key press to select the

$$
=T_{d m(o p)}+T_{k}
$$

Putting the value of $T_{d m}$ in (3) we get,

$T=T_{h}+K_{e f f} \cdot T_{k}+w \cdot d \cdot T_{m}+K_{e f f} \cdot T_{d m(o p)}+w \cdot T_{k}$

For present scenario, we can neglect $T_{h}$ as it is of no use. $T_{k}$ is also modified to take into account the search time of a key to be pressed in the on-screen keyboard.

From table 1 it is evident that layout 1 or $2, t_{S}=$ time for finding any key and moving focus (1.77/1.73s) needs to be considered. Thus equation (4) becomes

$$
\begin{aligned}
T= & K_{e f f}\left(T_{k}+t_{s}\right)+w \cdot d \cdot T_{m} \\
& +K_{e f f} \cdot T_{d m(o p)}+w\left(T_{k}+t_{s}\right)
\end{aligned}
$$

This equation can be used to evaluate the dynamic mental operator $T_{d m(o p)}$ which takes into account the additional cognitive load of the users.

\section{User Studies Conducted}

Four separate user studies were conducted for evaluating the onscreen keyboard.

User study 1: Study to evaluate the performance of initial keyboard layout

After finalizing the initial onscreen keyboard shown in figure 1, a user study was conducted to gather us er feedback on the proposed layout. Multiple techniques were employed to gather feedback from different users with a view to discover the strengths and weaknesses of the current design and implementation. The first set of user studies involved two aspects - a) Comparison of a standard QWERTY on-screen keyboard layout with the proposed layout, and b) User Perception Study for the proposed layout for finding out imp rovement areas. 
Users: 25 users were selected from different age group having different keyboard exposure, however the scope of the evaluation was kept limited to finding average user response and detailed demography based study was kept out of scope.

Tasks: The users were asked to type particular short message service (SMS) text, Email and uniform resource locator (URL)texts on the containing STB using a standard QWERTY on-screen keyboard and the proposed on-screen keyboard. Users were asked to type the text "The quick brown fox jumps over a lazy dog" on SMS and Email applications. This particular sentence was used because it contains all the alphabets in English. Users were also asked to type "www.google.com" as the URL in the Internet Browser Application. The time taken to type was noted.

These users were also asked to get familiar with the proposed on-screen keyboard via practice and same data were recorded after they became conversant. User perception study

A separate study was conducted on how the new on-screen keyboard was perceived by users. A user set of 25 respondents (same as used for user study 1). They were approached with a set of simple questions given below. Users were requested to provide a score on a scale of 1 to 5 where 1 indicated the poorest experience, and 5 indicated the best experience.

User perception study questions:

1. Does the on-screen keyboard provide enough assistance?

2. How is the ease of use of the on-screen keyboard?

After capturing the responses from a set of 25 respondents, the results were analysed. Based on the results obtained some modifications were adopted for the onscreen keyboard layout.

User study 2:Study to find out KLM-GOMS operator values

The pointing operator of original KLM-GOMS model has been modified keeping in mind the present scenario. Here we have re-defined $\mathrm{P}$ as the total time taken in finding a key on a particular layout and moving the focus to select the block containing that particular key. To estimate the value of $\mathrm{P}$, a user study was conducted. A group of 20 users of different age group were selected for the study. They were given three different layouts (layout1, 2 and QW ERTY) one at a time.

Tasks:During the study, a tape recorded message consisting of 20 randomly selected alphabets was played. The users were instructed to focus on the particular block containing the alphabets. The time taken to fin ish the session was noted using a stop watch. To reduce the error as much as possible, the average value was taken for each user. For two different layouts the values obtained are $1.77 \mathrm{~s}$ (for layout 1 ) and $1.73 \mathrm{sec}$ (for layout 2).The value of $\mathrm{P}$ for standard QWERTY keyboard layout has been adopted as $1.10 \mathrm{sec}$ as defined in original KLM model. The difference between these two values $(1.77 \mathrm{~s}$ and $1.73 \mathrm{~s})$ is due to the difference in alphabet distribution.

\section{User study 3 : Evaluation of word prediction feature}

A user study was conducted to gather feedback from different users with a view to discover the effect of usage of the prediction along with the onscreen keyboard.

Users: A group of 20 users of different age group were selected for the study. Half of these users were male and other half female respondents. 5 of them were very good typists, 10 had average typing speed, remaining 5 were slow typists.

Tasks: Six phrase sets were selected randomly from MacKenzie's test phrase set[12]. Users were given an initial familiarization phrase and then asked to enter six phrases at one go. Time taken by each user and the number of keystrokes required to type the phrase were recorded. In order to account for differing user effort, the main statistics used to measure speed was time per character - measured by the total time taken for each phrase divided by the length of the phrase as described in[13].

User study 4: Study to find out dynamic mental operator

A user study was conducted to find out the dynamic mental operator.

Users: A group selected for user study 3 was also asked to participate in user study 4.

Tasks:Six phrase sets were selected randomly from MacKenzie's test phrase set[12]. Users were given an initial familiarization phrase and then asked to enter six phrases at one go using predictive on-screen keyboard shown in figure 6 . Time taken by each user and the number of keystrokes required to type the phrase were recorded.

Dynamic mental operator was calculated using equation (5) for each user.

\section{Methodology for Design Evaluation using KLM-GOMS}

The KLM-GOMS operators used in the present scenario are given in table 1 . We have applied the concept of KLM-GOMS to evaluate the on- screen layouts for an email sending application. The KLM-GOMS for sending an email is shown below:

Table 2. KLM-GOMS for email sending application

\begin{tabular}{|c|c|}
\hline Goal & Sub goal \\
\hline \multirow{4}{*}{ Compose and send an email } & Open browser \\
\cline { 2 - 2 } & Open gmail server \& login \\
\cline { 2 - 2 } & Compose mail \\
\cline { 2 - 2 } & Dispatch \\
\hline
\end{tabular}

During the process of calculation and evaluation, some keyboard level operations were defined. These are actually the basic operations to be performed while using the layouts ( 1 and 2) with the remote control and thus served as the basic building blocks of the GOMS model. The time taken for these operations were calculated based on the KLM 
operators explained in table 1.The values are different for different layouts. The basic operations are given in table 3 .

Table 3. Basic operations of KLM-GOMS

\begin{tabular}{|c|c|c|c|}
\hline Operations & $\begin{array}{c}\text { Time for } \\
\text { layout1(fig 4) } \\
\text { in sec }\end{array}$ & $\begin{array}{c}\text { Time for } \\
\text { layout2(fig 5) } \\
\text { in sec }\end{array}$ & $\begin{array}{c}\text { Time for } \\
\text { QWERT Y in } \\
\text { sec }\end{array}$ \\
\hline $\begin{array}{c}\text { Open/close } \\
\text { onscreen } \\
\text { keyboard } \\
\text { layout. }\end{array}$ & 0.4 & 0.4 & 0.4 \\
\hline Find any key & 1.07 & 1.03 & 1.1 \\
\hline $\begin{array}{c}\text { Move focus to } \\
\text { select a key }\end{array}$ & 0.7 & 0.7 & 2.6 \\
\hline $\begin{array}{c}\text { Move finger to } \\
\text { the cornerkeys }\end{array}$ & 0.2 & 0.2 & 0.2 \\
\hline $\begin{array}{c}\text { Enter a } \\
\text { character using } \\
\text { keyboard }\end{array}$ & 2.17 & 2.13 & 4.0 \\
\hline
\end{tabular}

\section{Results of User Studies Conducted}

\subsection{Results of User Study 1}

In user study 1, we have compared our novel onscreen keyboard layout against the traditional QW ERTY layout.

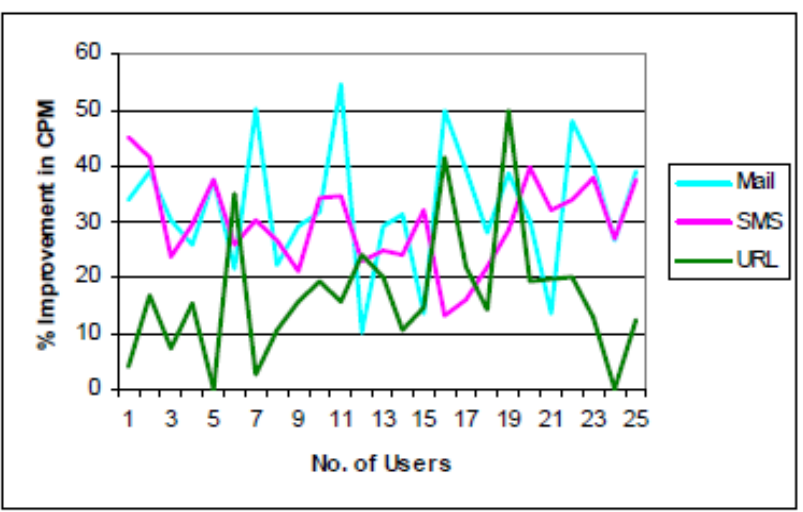

Figure 7. Improvement over on-screen QWERTY keyboard

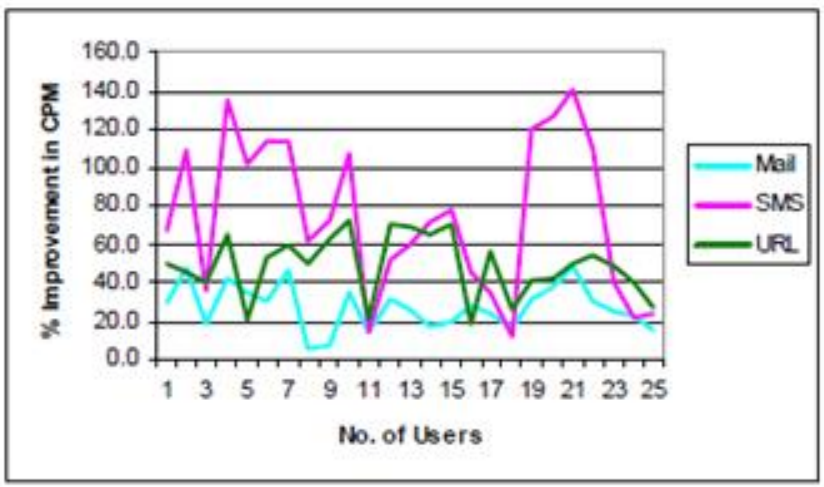

Figure 8. Improvements after practice

The measurements were done in terms of characters per minute (CPM). The CPM values were calculated for each user and were plotted for both standard QWERTY onscreen keyboard and for this new layout. The plots are shown in figure 7. It can be observed from the plots that there are significant improvements in CPM for the proposed layout over the QWERTY one indicating that the proposed layout is better. The average percentage improvement using the proposed keyboard over QWERTY on-screen keyboard is $27 \%$ for Email, $38 \%$ for SMS, and $41 \%$ for URL typing. The graph in Figure 7 displays the percentage imp rovement in CPM for all the users for each of the use cases like Email, SMS and URL.

Figure 8 shows the results after some practice compared to the initial runs. It shows an average improvement of $31.8 \%$ for email, $29.9 \%$ for SMS and $16.69 \%$ for URL typing. Detailed analysis of the results is given in[10].

\subsection{Results of User Study 2}

User study 2 was conducted to estimate the value of pointing operator (P) of the extended KLM-GOMS model.

For layout 1the value of $\mathrm{P}$ operator estimated was $1.77 \mathrm{~s}$ and that for layout 2 was $1.73 \mathrm{sec}$. The value of $\mathrm{P}$ for standard QWERTY keyboard layout has been adopted as $1.10 \mathrm{sec}$ as defined in original KLM model. The difference between these two values $(1.77 \mathrm{~s}$ and $1.73 \mathrm{~s})$ is due to the difference in alphabet distribution in two layouts. The details of the results are given in[11].

\subsection{Results of User Study 3}

User study 3 was conducted to evaluate the usage of the predictive onscreen keyboard over non-predictive text entry.

We have calculated percentage (\%) improvement defined as ratio of decrease in typing time with prediction for a particular string to that without prediction for the same string. We have also calculated the Words per minute (WPM), defined as the number of words entered per minute. To calculate this, we counted the total number of letters (including spaces) present in a phrase. Dividing the number of characters by 5 we get "words" and dividing time taken in sec. by 60 yields "per min." [14].

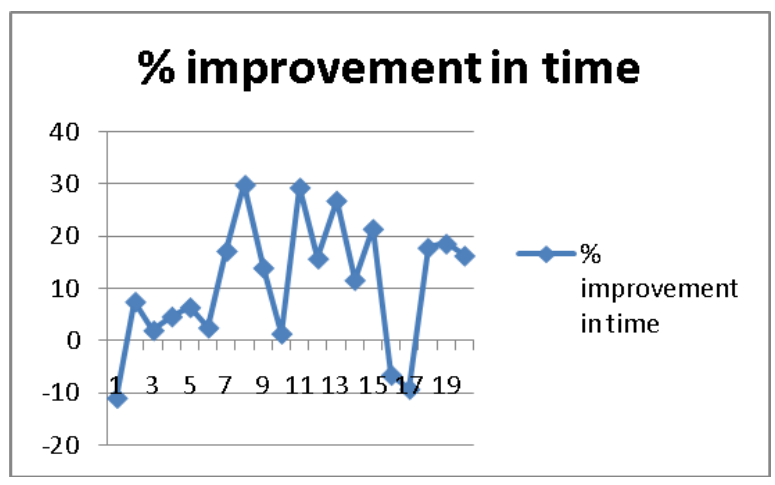

Figure 9. Percentage improvements in time

The improvement in time is shown in fig 9.The average improvement is $10 \%$. As seen from the graph, the $\%$ improvement value degraded for three users. According to them, every time they entered a character, it was difficult for them to scan through the suggested words and select one. 
According to them, the word completion feature interfered with their thought process and effectively delayed the typing process. Instead they felt it easier to type using the standard remote control which does not incorporate any word completion feature.

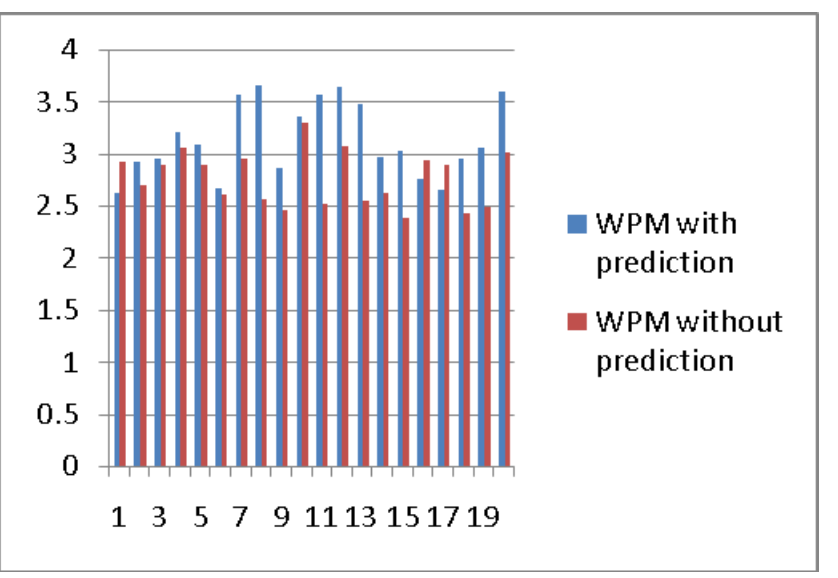

Figure 10. WPM with and without prediction

Figure 10 shows the WPM analysis results for typing with and without prediction.

The WPM value increases subsequently for almost all users when they enabled prediction and typed. For three users the WPM value was less with prediction for the reasons mentioned above.

\subsection{Results of User Study 4}

Using predictive onscreen keyboard imposes an additional cognitive load on the user as explained in section 4. A separate user study was conducted to find out the value of dynamic mental operator for predictive text entry.

Time taken for typing the test phrases and the effective key presses for each user are shown in fig 11 below.

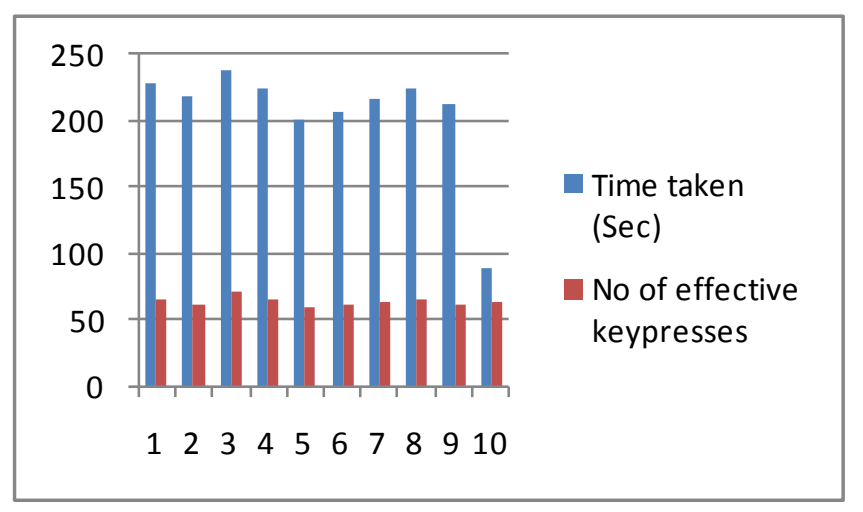

Figure 11. Time taken and no of key presses for different users

Based on the data obtained through user study, the dynamic mental operator $\left(T_{d m(o p}\right)$ was calculated using equation (5).From table 1 we get,

$t_{s}=$ time for finding any key and moving focus $=1.77 \mathrm{~s}$

$T_{k}=$ time taken for button press $=0.02 \mathrm{~s}$

$T_{m}=$ Mental preparation and thinking time $=1.35 \mathrm{~s}$

Values of $\mathrm{w}, K_{\text {eff }}$ and total time taken for typing are different for different users and are given in table 4 below.
The mental operator for different users is given in table 4 below. The average value of $T_{d m(o p)}$ was found to be 0.063 .

Table 4. Dynamic mental operator for different users

\begin{tabular}{|c|c|c|c|c|}
\hline User & $\begin{array}{c}\text { Totaltime } \\
\text { taken to } \\
\text { type in } \\
\text { sec }\end{array}$ & $\begin{array}{c}\text { Effective } \\
\text { key-presses }\left(K_{\text {eff }}\right)\end{array}$ & $\begin{array}{c}\text { No of } \\
\text { words } \\
\text { typed } \\
(\mathrm{w})\end{array}$ & $\begin{array}{c}\mathrm{T}_{\mathrm{dm}(\mathrm{op})} \\
\mathrm{Sec}\end{array}$ \\
\hline 1 & 228.62 & 65.43 & 30 & 0.0935 \\
\hline 2 & 218.66 & 61.84 & 29 & 0.102 \\
\hline 3 & 237.40 & 72.61 & 30 & 0.010 \\
\hline 4 & 224.28 & 65.43 & 30 & 0.027 \\
\hline 5 & 200.26 & 59.85 & 25 & 0.074 \\
\hline 6 & 206.14 & 61.84 & 26 & 0.0515 \\
\hline 7 & 216.98 & 63.84 & 28 & 0.064 \\
\hline 8 & 224.5 & 65.43 & 30 & 0.035 \\
\hline 9 & 212.52 & 63.04 & 27 & 0.0648 \\
\hline 10 & 215.88 & 63.84 & 28 & 0.049 \\
\hline
\end{tabular}

\subsection{Comparison of Results of KLM-GOMS and that Obtained through User Study}

For normal text entry, the user study 1 and 2 reveals that the layouts $1 \& 2$ allow much faster text entry compared to onscreen QWERTY layout[9],[10]. The percentage improvement in time for layouts $1 \& 2$ over onscreen QWERTY layout was approximately $44.23 \%$ and $45 \%$ respectively (shown in table 5). This is due to the fact that in layouts $1 \& 2$, four alphabets have been combined in a single block. The percentage improvement for layouts 1 over layout 2 was found to be approximately $2 \%$.Though both of them have similar blocked structure, the difference arises due to the difference in time required to enter a character for two layouts.

To validate this, we have applied extended KLM-GOMS modelling on different layouts., We set up the scenario of sending an email and modelled it with our parameters. The time taken for different sub-goals of email sending application is shownin table 5below. The values tabulated were obtained by decomposing sub-goals further until operator level tasks are reached. The operator level tasks are given in table 3

Table 5. Timetaken for different keyboard layouts

\begin{tabular}{|c|c|c|c|}
\hline Sub-goals & $\begin{array}{c}\text { Time for } \\
\text { layout 1 in sec }\end{array}$ & $\begin{array}{c}\text { Time for layout } \\
\text { 2 in sec }\end{array}$ & $\begin{array}{c}\text { Time for } \\
\text { QWERT Y in } \\
\text { sec }\end{array}$ \\
\hline $\begin{array}{c}\text { Open } \\
\text { browser }\end{array}$ & 0.5 & 0.5 & 0.5 \\
\hline $\begin{array}{c}\text { Open gmail } \\
\text { server \& } \\
\text { login }\end{array}$ & 45.3 & 44.5 & 82.1 \\
\hline $\begin{array}{c}\text { Compose } \\
\text { mail }\end{array}$ & 68.03 & 65.87 & 115.1 \\
\hline Dispatch & 0.4 & 0.4 & 0.4 \\
\hline
\end{tabular}

Hence based on cognitive models, it is found that the improvement (shown in table 6) of the layout shown in fig 1 over QWERTY layout is approximately $45.75 \%$ and that for the layout shown in fig 2 over QW ERTY is $46.75 \%$. It 
also shows that the \% improvement of layout 2 over layout 1 is approximately $1 \%$. Thus the keyboard shown in layout 2 is faster than that shown in layout 1 . Thus the results of the user study matches with the theoretical values obtained through KLM-GOMS modelling.

Table 6. Comparison of different layouts

\begin{tabular}{|c|c|c|}
\hline Layouts & $\begin{array}{c}\text { \% improvement in } \\
\text { typing speed from } \\
\text { user study }\end{array}$ & $\begin{array}{c}\text { \% improvement in } \\
\text { typing speed from } \\
\text { KLM-GOMS }\end{array}$ \\
\hline $\begin{array}{c}\text { Layout 1 over } \\
\text { QWERTY }\end{array}$ & 44.23 & 45.75 \\
\hline $\begin{array}{c}\text { Layout 2 over } \\
\text { QWERTY }\end{array}$ & 45 & 46.75 \\
\hline $\begin{array}{c}\text { Layout 2 over } \\
\text { layout1 }\end{array}$ & 2 & 1.00 \\
\hline
\end{tabular}

\section{Design Modifications basedon User Study}

Based on the results obtained from user study 1, some changes were adopted in the initial keyboard layout. These changes are:

(i ) Layout of alphabets: In the initial release of the new keyboard, the letters ' $a, b, c, d$ ' were in the same block with ' $a$ ' and 'b' in the upper half and 'c'and' $d$ ' in the lower half. However during usability reviews, majority of the users pointed out that they would be more comfortable to have the letters ' $a$ ', 'b', 'c', 'd', 'e', ' $f$ ' in the same horizontal line as long as it possible, and once the space runs out they want to look in the next horizontal line. Accordingly the layout of the keyboard was changed to that shown in figure 4.

(ii) Most frequently used Smileys: Initial on-screen keyboard had a number of smileys which were selected after discussion among the team members. Later on the most frequently used smileys were updated to accommodate users' preferences. The smileys are included in the symbol page as shown in Figure 12.

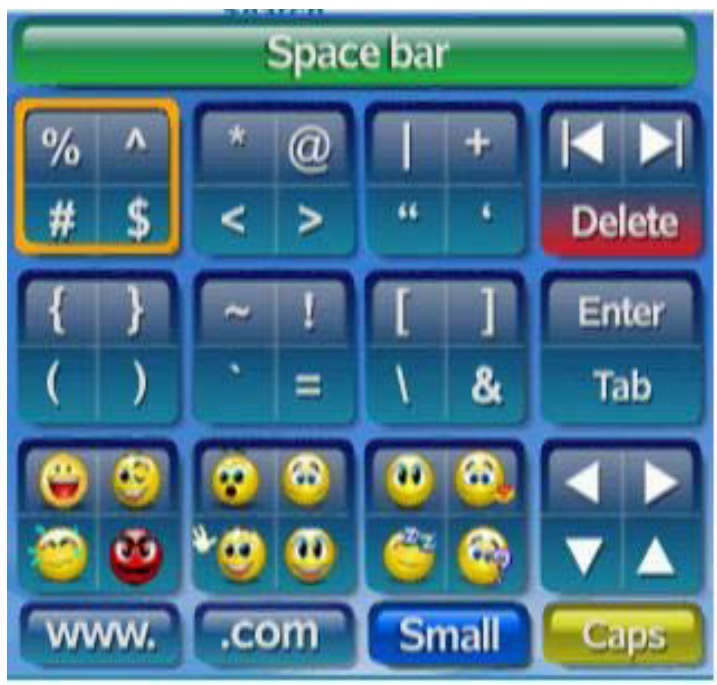

Figure 12. Symbols and Smileys (iii) The missing @:In the very beginning the symbol '@' was not available in the frequently used symbols in the main layout. During the user study, users complained about this is sue while typing emails. Based on that the symbol '@) was included in the frequently used symbols in the main layout

(iv) Hot keys to speed up typing: Number of users suggested giving some additional options as hot keys so that some of the most commonly used requirements can be expedited. Accordingly different users' preferences for hot keys were noted and analysed along with the constraint of real estate space on the accompanying remote control. 4 colour keys on the remote were decided to be used as hot keys - green for typing "space", red for typing "delete", yellow and blue to bring up the capital/small letter screen or the symbol screen based on the keyboard context at a particular instance on the remote control.

(v) Visual effect of key press: During the study users pointed out that they were not sure or did not know which key has been indeed selected and typed. This at times led user to type a desired letter multiple number of times. As a consequence, it was felt that user should get some kind of visual indication on the selected character itself. Accordingly the effect of key press was introduced. As shown in Figure13, when the letter " $A$ " is selected, its appearance mo mentarily changes to highlight it distinctly.

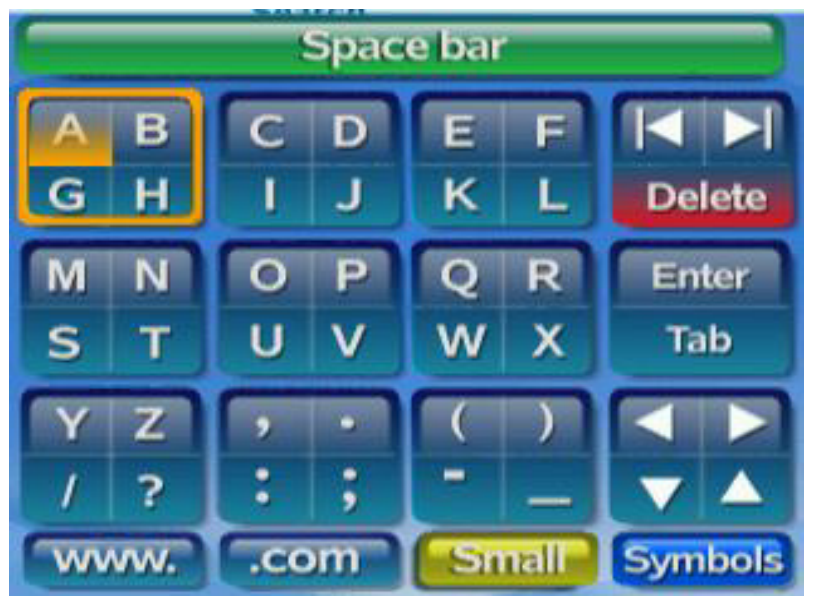

Figure 13. Visual effects of key-press and colored hot keys

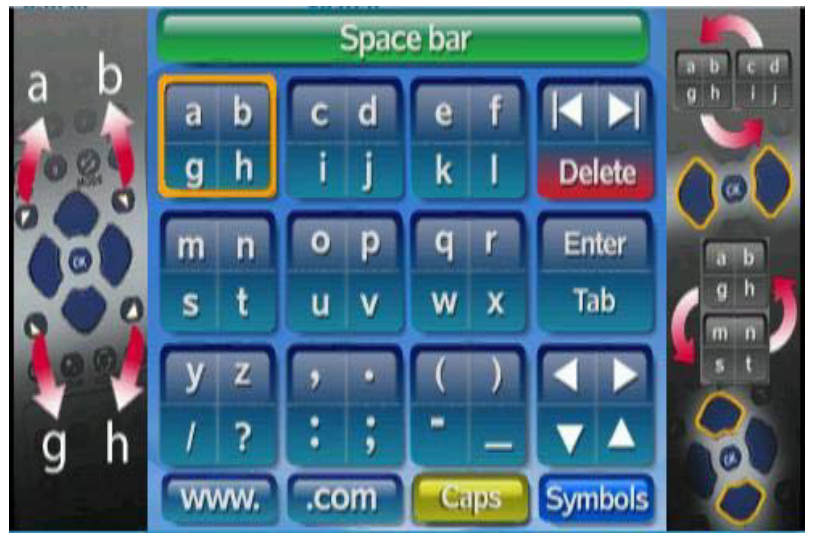

Figure 14. Co-located help 
(vi ) Need for co-located help:The user experience and perception study conducted before beta launch showed satisfactory result, but the consumer feedback received was not that good. Initially the users have difficulty in figuring out how to use the proposed on-screen keyboard, but combined with the encouraging findings from the previous section, it can be deduced that, once users are initiated to the mechanis m of use of this new keyboard, they can type faster with it. So as an aid to the users, a brief and crisp pictorial help as shown in Figure 14 were embedded along with the on-screen keyboard informing users about how to use it.

\section{Conclusions}

This paper elaborates the mechanism for hierarchical navigation and selection of characters and symbols on an onscreen keyboard which require reduced number of key strokes. This work makes accessing services like Internet browsing, email, SMS, Instant messenger (IM) from TV easier, whichrequires less effort from user, thereby enhancing the user experience. After initial design was done and implemented, extensive user study isdone for taking the user feedback.

User study 1 shows that the average percentage improvement using the proposed keyboard over QWERTY on-screen keyboard is $27 \%$ for Email, $38 \%$ for SMS, and $41 \%$ for URL typing.After some initial practice, afurther improvement of $31.8 \%$ for email, $29.9 \%$ for SMS and $16.69 \%$ for URL typingis obtained.

User study 2 is conducted to estimate the parameter values for KLM-GOMS model. Based on cognitive models, it is found that the improvement (shown in table 6) of the layout shown in fig 1 over QWERTY layout is approximately $45.75 \%$ and that for the layout shown in fig 2 over QWERTY is $46.75 \%$. It also shows that the $\%$ improvement of layout 2 over layout 1 is approximately $1 \%$. Thus the keyboard shown in layout 2 is faster than that shown in layout 1 .

User study 3 is conducted to compare usage of the predictive onscreen keyboard over non-predictive text entry.The average improvement is $10 \%$ except for three users for which the layout gives a degraded performance.

User study 4 is conducted to estimate the value of dynamic mental operator.The additional cognitive load imposed on the user while using predictivetext entry, gives rise to this parameter. The average value of dynamic mental operator is found to be $0.063 \mathrm{sec}$.

Some modifications areintroduced based on the user feedback collected. Based on the results obtained and tabulated above, we can conclude that the extended KLM-GOMS model used here can efficiently predict the performance of the onscreen keyboard layout. The results obtained through user study and the values predicted by KLM GOMS model match with each other. Hence this particular onscreen keyboard layout can be used and operated efficiently with a remote control device for text entry on television and set-top box applications.

In future we plan to evaluate the cognitive load of predictive onscreen keyboard using an EEG device.

\section{ACKNOWLEDGEMENTS}

Authors would like to convey their sincere acknowledgement to the individuals who participated in the various user studies and made this project a reality.

\section{REFERENCES}

[1] World Internet Usage Statistics News and World Population Stats.

[2] http://www.internetworldstats.com/stats.htm.

[3] ITU report. Measuring the information society $2011 \mathrm{p}$ 108-128.

[4] Technical report. I-cube $2009-2010$ by IMRB

[5] William Cooper, The interactive television user experience so far, Proceeding of the 1st international conference on Designing interactive user experiences for TV and video, October 22-24, 2008, Silicon Valley, California, USA

[6] Geleijnse, G., Aliakseyeu, D., and Sarroukh, E. 2009. Comparing text entry methods for interactive television applications.Proceedings of the Seventh European Conference on European interactive Television Conference (Leuven, Belgium, June 03 - 05, 2009). EuroITV '09. ACM, New York, NY, 145-148

[7] Iatrino and S. Modeo. Text editing in digital terrestrial television: a comparison of three interfaces. In Proceedings of EuroITV'06, Athens, Greece,2006

[8] Ingmarsson, M., Dinka, D., Zhai, S., TNT-A numeric keypad based text input method, Proc. CHI 2004: ACM Conference on Human Factors in Computing Systems. Vienna, Austria. CHI Letters 6(1), 639-646,ACM Press.

[9] Holleis, P., Otto, F., Hussmann, H., and Schmidt, A. (2007). "Keystroke-level model for advanced mobile phone interaction," in CHI '07: Proceedings of the SIGCHI conference on Human factors in computing systems, pages 1505-1514, New York, NY, USA.

[10] Pal, A., Bhaumik, C., Kar, D., Ghoshdastidar, S., Shukla, J., A novel on-screen keyboard forhierarchical navigation with reduced number of key strokes, IEEE International Conference on Systems, Man and Cybernetics, 2009. SMC 2009.

[11] Pal, A., Chatterjee, D., Kar, D., Evaluation and Improvements of on-screen keyboard for TV and set -top box, 15th IEEE International symposium on Consumer Electronics, June 2011, Singapore

[12] Basu, A, Chatterjee, D.,Pal, A., Extended KLM-GOMS modeling and Evaluation of onscreen keyboard layouts for television based internet applications, accepted in 4th 
international conference on Human Computer Interaction , Pune, India, April 2012

[13] Holleis, P., Otto, F., Hussmann, H., and Schmidt, A. (2007) "Keystroke-level model for advanced mobile phone interaction," in CHI '07: Proceedings of the SIGCHI conference on Human factors in computing systems, pages 1505-1514, New York, NY, USA.

[14] MacKenzie, I. S., and Soukoreff, R. W. "Phrase sets for evaluating text entry techniques," in CHI '03 extended abstracts, Lauderdale, USA, 754-755, 2003.

[15] Dunlop, D, Mark, and Taylor, Finbarr, Tactile feedback for predictive text entry http://www.cis.strath.ac.uk/cis/research/ publications/papers/strath_cis_publication_2327.pdf

[16] MacKenzie, I. S. (2007). Evaluation of text entry techniques. In MacKenzie, I. S., and Tanaka-Ishii, K. (Eds.) Text entry systems: Mobility, accessibility, universality, pp. 75-101. San Francisco, CA: Morgan Kaufmann.

[17] Bhattacharya, S., Basu, A. \&Samanta, D. (2008). Computational Modeling of User Errors for the Design of Virtual Scanning Keyboards. IEEE Transactions on Neural Systems and Rehabilitation Engineering, 16(4), pp. 400-409.

[18] Bhattacharya, S., Basu, A. \&Samanta, D. (2008). Performance Models for Automatic Evaluation of Virtual Scanning Keyboards. IEEE Transactions on Neural Systems and Rehabilitation Engineering, 16(5), pp 510-519.
[19] Magnuson, T., Hunnicutt, S. "Measuring the Effectiveness of Word Prediction: The Advantage of Long-term Use "KTH Computer Science and Communication Journal: TMH-QPSR, volume 43, pages 057-067

[20] Koester, Heidi H, Levine, Simon. P. "Model Simulations for User Performance With Word Prediction" AAC Augmentative and Alternative Communication,0743-4618/98 Vol 14, March 1998.

[21] Dunlop, D., Mark, Crossan, Andrew "Predictive text entry methods for mobile phones". Personal Technologies, Vol 4, Numbers 2-3(2000), p- 134-143, DOI: 10.1007/BF01324120

[22] Trnka, K., McCaw, J., Yarrington, D., McCoy, K. F., and Pennington, C. 2009. User interaction with word prediction: The effects of prediction quality. ACM Trans. Access. Comput. 1, 3, Article 17 (February 2009), 34 pages. DOI = 10.1145/1497302.1497307.

[23] http://doi.acm.org/10.1145/1497302.1497307.

[24] Pal, Arpan and Prashant, M. and Ghose, Avik and Bhaumik, Chirabrata 2010. Home Infotainment Platform - A Ubiquitous Access Device for Masses. Ubiquitous Computing and Multimedia Applications. Communications in Computer and Information Science. Volume 75, pages 11-19. ISBN: 978-3-642-13467-8 